\title{
Comparison of Two-Phase Darcy's Law with a Thermodynamically Consistent Approach
}

\author{
Jennifer Niessner • Steffen Berg • \\ S. Majid Hassanizadeh
}

Received: 24 March 2010 / Accepted: 14 January 2011 / Published online: 5 February 2011

(C) The Author(s) 2011. This article is published with open access at Springerlink.com

\begin{abstract}
The extended Darcy's law is a commonly used equation for the description of immiscible two-phase flow in porous media. It dates back to the 1940s and is essentially an empirical relationship. According to the extended Darcy's law, pressure gradient and gravity are the only driving forces for the flow of each fluid. Within the last two decades, more advanced and physically based descriptions for multiphase flow in porous media have been developed. In this work, the extended Darcy's law is compared to a thermodynamically consistent approach which explicitly takes the important role of phase interfaces into account, both as entities and as parameters. In this theoretically derived approach, forces related to capillarity and interfaces appear as driving/resisting forces, in addition to the traditional terms. It turns out that the extended Darcy's law and the thermodynamically based approach are compatible if either (i) relative permeabilities are a function of saturation only, but capillary pressure is a function of saturation and specific interfacial area or (ii) relative permeabilities are a function of saturation and saturation gradients. Theoretical considerations suggest that the former alternative is only valid in case of reversible displacement while in the general case (irreversible displacement), the latter alternative is relevant.
\end{abstract}

Keywords Porous media $\cdot$ Two-phase flow $\cdot$ Interfacial area

Thermodynamically consistent approach

\footnotetext{
J. Niessner

Institute of Hydraulic Engineering, University of Stuttgart, 70550 Stuttgart, Germany

e-mail: Jennifer.Niessner@iws.uni-stuttgart.de
}

\section{S. Berg $(\bowtie)$}

Shell International Exploration and Production B.V., Kesslerpark 1, 2288 GS, Rijswijk (ZH),

The Netherlands

e-mail: steffen.berg@shell.com

\section{S. M. Hassanizadeh}

Department of Earth Sciences, Faculty of Geosciences, Utrecht University, P.O. Box 80021, 3508 TA Utrecht, The Netherlands

e-mail: hassanizadeh@geo.uu.nl 


\section{List of Symbols}

\section{Latin Variables}

$a_{\alpha \beta} \quad$ Specific interfacial area of $\alpha \beta$-interface $\left[\mathrm{m}^{-1}\right]$

$\underline{g} \quad$ Gravity $\left[\mathrm{m} \mathrm{s}^{-2}\right]$

$\bar{k}_{r \alpha} \quad$ Relative permeability of phase $\alpha[-]$

$p_{\alpha} \quad$ Pressure of phase $\alpha[\mathrm{Pa}]$

$p_{\mathrm{c}} \quad$ Capillary pressure $[\mathrm{Pa}]$

$t \quad$ Time [s]

$\underline{v}_{\alpha}, \underline{v}_{\alpha \beta} \quad$ Velocity of phase $\alpha$ or $\alpha \beta$-interface, respectively [ $\mathrm{m} \mathrm{s}^{-1}$ ]

A Area $\left[\mathrm{m}^{2}\right]$

$E_{d} \quad$ Net efficiency [-]

$H \quad$ Specific Helmholtz free energy $\left[\mathrm{m}^{2} \mathrm{~s}^{-1}\right]$

$\underline{K} \quad$ Intrinsic permeability tensor $\left[\mathrm{m}^{2}\right]$

$\overline{\bar{L}} \quad$ Length [m]

$Q_{\alpha} \quad$ External source or sink of phase $\alpha\left[\mathrm{s}^{-1}\right]$

$Q \quad$ Volumetric flux $\left[\mathrm{m}^{3} \mathrm{~s}^{-1}\right]$

$\underline{R} \quad$ Resistance tensor $\left[\mathrm{Pa} \mathrm{s} \mathrm{m}^{-2}\right.$ ]

$\bar{S}_{\alpha} \quad$ Saturation of phase $\alpha[-]$

$T_{\alpha} \quad$ Temperature of phase $\alpha[\mathrm{K}]$

$V_{b} \quad$ Bulk volume $\left[\mathrm{m}^{3}\right]$

$W \quad$ Work [J]

\section{Greek Symbols}

Symbol Meaning

$\gamma_{\alpha \beta} \quad$ Macroscopic interfacial tension [Pa m]

$\mu_{\alpha} \quad$ Dynamic viscosity of phase $\alpha$ [Pas]

$\phi \quad$ Porosity [-]

$\rho_{\alpha} \quad$ Density of phase $\alpha\left[\mathrm{kg} \mathrm{m}^{-3}\right]$

$\sigma_{\alpha \beta} \quad$ Pore-scale interfacial tension of $\alpha \beta$-interface [Pa m]

$\Gamma_{\alpha \beta} \quad$ Areal mass density of $\alpha \beta$-interface $\left[\mathrm{kg} \mathrm{m}^{-2}\right]$

$\Theta \quad$ Contact angle [rad]

\section{Subscripts}

c Capillary

$n \quad$ Non-wetting

$w \quad$ Wetting

$\alpha \quad$ Phase

$\alpha \beta \quad$ Interface

\section{Introduction}

Fluid flow in porous media is a subject of general relevance in science, technology, and environment. Classical applications comprise petroleum engineering where oil and gas are 
produced from the porous sedimentary rock, civil engineering where stability issues of geotechnical systems are investigated, or environmental engineering where flow and transport of contaminants in the subsurface are considered. Recently, in the field of flow and transport in porous media, new topics have evolved. They range from storage of carbon dioxide in deep geological formations and the migration of methane emitted by abandoned coal mines to paper manufacturing, processes in polymer electrolyte membrane fuel cells, to fluid flow processes in the human body (Huyghe et al. 1989; Vankan et al. 1996). While in many cases only a single phase is mobile, there is a large number of cases of practical relevance where two or more phases flow at the same time with the case of two-phase flow being the simplest case of multi-phase flow.

\subsection{Approaches for Two-Phase Flow in a Porous Medium}

The most commonly used description for macro-scale two-phase flow in porous media uses a phenomenological extension of Darcy's law introducing the saturation-dependent parameter relative permeability. The introduction of an additional parameter, capillary pressure becomes necessary for the closure of the system. However, there are a number of well-known problems associated with this model (Hassanizadeh and Gray 1993a,b) which are addressed in more detail in Sect. 2.1.3. In brief, first, constitutive relationships (mainly capillary pressure-saturation, but also relative permeability-saturation relations) are found to be hysteretic (Killough 1976) indicating that the set of independent parameters may be incomplete. Second, it is well known that depending on the system considered, there may be more driving forces for the flow of phases than are included in Darcy's law (saturation gradients, temperature gradients, gradients in chemical potential,...). Third, dynamic pressure effects are found to be important in case of transient flow (Stauffer 1978; Hassanizadeh and Gray 1990, 1993b; DiCarlo 2004; Bottero et al. 2006; Mirzaei and Das 2007; DiCarlo 2005; Manthey et al. 2005). Finally, given the fact that the rate of interphase mass or heat transfer must depend on the amount of interfacial area, kinetic interphase mass and heat transfer can be modeled in a physically based way only if interfacial areas are included as parameters, like in the approach of Niessner and Hassanizadeh $(2009, c)$. The absence of interfacial area as a parameter in classical models makes it impossible to account for the above-mentioned processes in a physically based way. These shortcomings obviously occur as a consequence of making empirical extensions to an empirical law without taking thorough account of all the physical principles involved like the laws of thermodynamics and the omnipresence of phaseinterfaces.

In the past decades, several approaches have been made to describe two-phase flow in a thermodynamically consistent way, partially also including interfacial areas as parameters. Among these are a rational thermodynamics approach by Hassanizadeh and Gray (1980, 1990, 1993a,b), a thermodynamically constrained averaging approach by Gray and Miller (2005) which is based on the work of Hassanizadeh and Gray (1990), mixture theory (Bowen 1982) and an approach based on averaging and non-equilibrium thermodynamics by Marle (1982) and Kalaydjian (1987). While Marle (1982) and Kalaydjian (1987) developed their set of constitutive relationships phenomenologically, Hassanizadeh and Gray (1990, 1993a), Gray and Miller (2005), Miller and Gray (2005), Bowen (1982) exploited the entropy inequality to obtain constitutive relationships. The authors of Hassanizadeh and Gray (1990, 1993a), Gray and Miller (2005), Miller and Gray (2005), Bowen (1982) all assume free energies to 
depend on saturation and thus, come up with an additional driving force in Darcy's law which depends on the saturation gradient.

\subsection{Purpose of this Work}

The extended two-phase Darcy description is used frequently by researchers and engineers and a large set of experimental data and parameters is available for a broad range of materials and applications. Despite the obvious shortcomings, it is still the most commonly used approach today and implemented in most numerical modeling software. Given the strengths of thermodynamically consistent and interfacial-area-based approaches and their potential importance for describing relevant problems like $\mathrm{CO}_{2}$ sequestration, it is now a pertinent question how they relate to the two-phase Darcy description and/or under which conditions the description of flow is equivalent in both approaches. Even though the commonly used two-phase Darcy flow description is phenomenological in nature and does not contain all driving forces for flow, it may still describe the relation between phase fluxes and pressure gradients correctly. As a consequence, the large set of published data on material parameters may be valid and after appropriate "translation" be useful in thermodynamically based theories as well. For that purpose, a knowledge of the relationships between parameters of the extended two-phase Darcy approach and the thermodynamically based approach is required. For example, Hassanizadeh and Gray (1990) conjectured that the dependency on saturation gradients is lumped into relative permeability. This means that relative permeability may contain contributions from other "hidden" variables like saturation gradients and possibly also interfacial area. Therefore, in this work, the extended two-phase Darcy model is directly compared to the approach of Hassanizadeh and Gray (1990, 1993a).

\subsection{Outline of this Work}

In Sect. 2, we first summarize the classical two-phase flow approach and then review the thermodynamically consistent approach for two-phase flow in porous media that considers phase-interfaces as separate entities (Hassanizadeh and Gray 1990, 1993a). In Sect. 3, the two theories are compared directly demanding equality of the specific phase fluxes which results in two possible cases which are investigated in detail. We find that either (i) relative permeabilities are a function of saturation only, but capillary pressure is a function of saturation and specific interfacial area or (ii) relative permeabilities are a function of saturation and saturation gradients.

\section{Mathematical Models for Two-Phase Flow in Porous Media}

\subsection{Classical Models}

For various applications mentioned in Sect. 1, it is impossible to resolve the porous medium and the relevant flow and transport processes in detail; the considered systems are much too large to allow for a detailed resolution of the pore space and the relevant processes. Instead, classical models usually proceed by describing the relevant processes on a volume-averaged scale. Therefore, a so-called representative elementary volume (REV) is identified for which volume-averaged quantities are provided. The standard procedure is to formulate continuity equations based on mass balances for each fluid phase $\alpha$ (here assumed incompressible) with respect to an REV: 


$$
\phi \frac{\partial S_{\alpha}}{\partial t}+\nabla \cdot \underline{v}_{\alpha}=Q_{\alpha},
$$

where $\phi$ is porosity (ratio of pore space to the volume of an REV), $S_{\alpha}$ is the saturation (fraction of pore space in an REV occupied by fluid $\alpha$ ), $\underline{v}_{\alpha}$ is the so-called extended Darcy velocity, and $Q_{\alpha}$ an external source or sink of the $\alpha$-phase. An important variable in these continuity equations is the extended Darcy velocity $\underline{v}_{\alpha}$. It is commonly prescribed by an empirical extension of a relationship that was postulated based on experimental observations by Darcy (1856) for one-dimensional single-phase flow through a homogeneous and isotropic porous medium. Therefore, in the following, we proceed by reviewing Darcy's law for single-phase flow, present its extension to two-phase flow, and discuss possible limitations.

\subsubsection{Single-Phase Flow and Darcy's Law}

Historically, Darcy's law was introduced in a phenomenological way for single-phase flow through sand. According to Darcy's law, the flux of a single phase is given by

$$
Q=A \frac{K}{\mu} \frac{\Delta p}{L},
$$

where $Q$ is the flux, $A$ is the cross section, $K$ the intrinsic permeability of the medium, $\mu$ the viscosity, and $\Delta p$ the pressure drop over sample length $L$ (Dullien 1992). The Darcy velocity is based on the flux and defined as $v=q=Q / A$. Darcy's law was later generalized to three-dimensional flow:

$$
\underline{v}=-\frac{\underline{\underline{K}}}{\mu} \cdot(\nabla p-\rho \underline{g})
$$

where $\nabla p$ is the pressure gradient and $\underline{K}$ is the intrinsic permeability tensor assumed to be a property of the porous solid only and $\underline{\bar{g}}$ is the gravity vector.

The same functional relationship between pressure gradient and flow rate has been found rigorously by homogenizing the Navier-Stokes equations (see Auriault 2005; Bear and Bachmat 1990), but through imposing the assumptions that inertial forces and friction within the fluid can be neglected. Hassanizadeh and Gray (1980) showed that Darcy's law may actually result from the macro-scale momentum balance equation by upscaling of pore-scale mass, momentum, energy, and entropy balances in the framework of a thermodynamic averaging theory and applying a large set of assumptions.

\subsubsection{Two-Phase Extension of Darcy's Law}

For multi-phase flow, the situation is conceptually more complicated. The presence of a second phase can, depending on its distribution in the porous network, hinder the flow of the first phase. Various attempts have been made at describing two-phase flow in porous media where the extension of Darcy's law from Eq. 3 is a very commonly used approach. While the principle functional relationship as in the single-phase Darcy's law is maintained, coefficients are generally made phase-specific and permeability is made saturation dependent.

In this phenomenological two-phase extension of Darcy's law, the specific flux of phase $\alpha(\alpha=w$ for the wetting phase and $\alpha=n$ for the non-wetting phase) is described as

$$
\underline{v}_{\alpha}=-\frac{\underline{\underline{K}} \alpha}{\mu_{\alpha}} \cdot\left(\nabla p_{\alpha}-\rho_{\alpha} \underline{g}\right),
$$


where $\underline{\underline{K}} \alpha$ is the saturation dependent two-phase permeability of the $\alpha$-phase. Although this extension of Darcy's law is empirical, this approach has been established as the standard approach for calculating specific fluxes in porous media.

By introducing the concept of relative permeability, the two-phase permeabilities are separated into products:

$$
\underline{\underline{K}}_{\alpha}=k_{r \alpha} \underline{\underline{K}}
$$

where $k_{r w}=k_{r w}\left(S_{\mathrm{w}}\right)$ and $k_{r n}=k_{r n}\left(S_{\mathrm{n}}\right)$ are the saturation-dependent relative permeabilities. $S_{\mathrm{w}}$ and $S_{\mathrm{n}}$ are the saturations of the wetting and non-wetting phases, respectively, and are constrained via

$$
S_{\mathrm{w}}+S_{\mathrm{n}}=1 .
$$

Due to this relationship, saturation-dependent functions are often expressed via the wettingphase saturation $S_{\mathrm{w}}$. The full set of equations describing two-phase flow consists of a mass balance equation (reduced to a volume balance, see Eq. 1) for each of the two fluid phases $\alpha$ where velocities are calculated using the extended form of Darcy's law given by Eq. 4, and restricted by the condition in Eq. 6. However, inserting the Darcy velocities into the volume balance equations results in three equations for four unknown quantities: two pressures $p_{\alpha}$ and the two saturations $S_{\alpha}$. In order to close the system of equations, it has been postulated that the difference in phase pressures is a function of the wetting-phase saturation. This postulation was based on observations that there is obviously some connection between pressure difference and saturation. By drawing an analogy to equilibrium considerations at a fluid-fluid interface, this pressure difference was called capillary pressure $p_{\mathrm{c}}$ :

$$
p_{\mathrm{c}}:=p_{\mathrm{c}}\left(S_{\mathrm{w}}\right):=p_{\mathrm{n}}-p_{\mathrm{w}},
$$

where it is assumed that the capillary pressure is a function of the wetting-phase saturation only.

\subsubsection{Known Limitations of the Two-Phase Darcy Approach}

In this work, we restrict ourselves to situations where for single-phase flow, Darcy's law is valid, i.e., flow in porous media at low Reynolds numbers $R e<1$ (so-called creeping flow). Extensions for larger Reynolds numbers like the Forchheimer equation (Hassanizadeh and Gray 1987) or high-porosity flow modeled by the Darcy-Brinkman equation (Brinkman 1947) are not considered here. In the following, a number of fundamental shortcomings of the conventional two-phase Darcy flow approach are listed:

- In the classical two-phase flow model, parameters like relative permeability and capillary pressure depend on saturation only. Observations like capillary pressure hysteresis, where the capillary pressure depends not only on saturation but also on saturation history (Killough 1976), provide direct evidence that the parameter set of the two-phase Darcy flow description is incomplete.

Theoretical work (Hassanizadeh and Gray 1990, 1993a,b), numerical modeling (JoekarNiasar et al. 2008, 2009; Niessner and Hassanizadeh 2008), and experiments (Culligan et al. 2004; Chen et al. 2007) have demonstrated that hysteresis (almost) disappears if the specific fluid-fluid interfacial area is introduced as a new parameter. This can be considered as an established view (Oostrom et al. 2001; Dalla et al. 2002; Gladkikh and Bryant 2003; Culligan et al. 2006; Seth and Morrow 2007). 
- Classically, macro-scale capillary pressure is assumed to be equal to the difference in nonwetting and wetting-phase pressures (Eq. 7). In dynamic situations, however, $p_{\mathrm{n}}-p_{\mathrm{w}}$ and $p_{\mathrm{c}}$ differ (Stauffer 1978; DiCarlo 2004; Bottero et al. 2006) by a term which depends on the time rate of change of saturation, $\partial S_{\mathrm{w}} / \partial t$ (Hassanizadeh and Gray 1990, 1993b; Hassanizadeh et al. 2002; Das et al. 2007).

- Viscous coupling between fluid phases is ignored (Ayub and Bentsen 2005, 1999; Zhang et al. 2008; Huang and Lu 2009). From a fluid mechanics point of view, at the interface between two immiscible fluids, the tangential stress is continuous. As a consequence, the two-phase Darcy description given in Eq. 4 would require "cross coupling" terms (Hassanizadeh and Gray 1993a). While this effect is in principle present for any flow situation (Zhang et al. 2008), its magnitude varies from situation to situation. It is found to be most significant in countercurrent flow (Eastwood and Spanos 1991).

- Relative permeabilities were introduced as parameters for scaling saturated permeability such that $0 \leq k_{r \alpha} \leq 1$. However, various cases with $k_{r \alpha}>1$ have been reported independently (see Berg et al. 2008 and references therein). This is supposedly caused by the lubricating effect of wetting films coating the rock surface during the flow of the non-wetting phase.

\subsection{Thermodynamic Averaging Approach}

In order to obtain a thermodynamically consistent set of equations, Hassanizadeh and Gray 1990 proceeded as follows:

1. Balance equations for mass, momentum, energy, and entropy for both fluid phases and phase-interfaces are formulated on the pore scale.

2. Pore-scale conservation equations are volume averaged over REVs to the macro scale (Gray and Hassanizadeh 1989).

3. Subsequently, the entropy production occurring in the macro-scale entropy balance equations is used to formulate the second law of thermodynamics (entropy inequality) on the macro scale.

4. The condition that the entropy has to reach its absolute minimum at equilibrium can be used to obtain constitutive relationships. Thus, constitutive relationships are not postulated, but derived as a result of the procedure.

Special attention was paid to the dependency of the Helmholtz free energies of phases and interfaces on the model parameters. It is commonly accepted that they depend on phase densities and temperature. To the best of our knowledge, it is also widely accepted that Helmholtz free energies of phases depend on saturation. Not including the dependence of phase or interface Helmholtz free energies on saturation leads to unacceptable results. For example, from the entropy inequality developed by Hassanizadeh and Gray (1990), it can be concluded that the neglect of the saturation dependence in the free energies leads, for isothermal systems, to the two phase pressures being equal, $p_{\mathrm{n}}=p_{\mathrm{w}}$. It is well known from experiments that this equality does not hold. For describing two-phase flow in a thermodynamic framework, researchers have generally included the saturation dependence of free energy; while Bowen (1982) made that assumption in the frame of mixture theory, Hassanizadeh and Gray (1990) made their investigations based on that dependency in a rational thermodynamics framework. Recently, Jackson et al. (2009) considered the approach of Hassanizadeh and Gray (1990) in the frame of a different thermodynamic approach also accounting for saturation dependency of the free energy. Another argument in favor of the saturation dependence of free energy concerns the work of drainage out- 
lined by Seth and Morrow (2007). They argue that the work $W$ done to the system (which partially leads to an increase in surface free energy) is parameterized by the area under the $p_{\mathrm{c}}\left(S_{\mathrm{w}}\right)$ curve at different saturations (Seth and Morrow 2007), i.e., $W=W\left(S_{\mathrm{w}}\right)$. Since the work of drainage represents an energy change in the system, it is a contribution to the Helmholtz free energy and therefore, Helmholtz free energy should depend on $S_{\mathrm{w}}$.

Hassanizadeh and Gray (1990) derived an equation for the Darcy velocity of phases:

$$
\underline{v}_{\alpha}=-\frac{-S_{\alpha}^{2} \underline{\underline{K}}_{\alpha}^{*}}{\mu_{\alpha}} \cdot\left(\nabla p_{\alpha}-\rho_{\alpha} \underline{g}+\rho_{\alpha} \frac{\partial H_{\alpha}}{\partial S_{\alpha}} \nabla S_{\alpha}\right),
$$

where $\underline{\underline{K}}_{\alpha}^{*}$ is the permeability tensor of phase $\alpha$.

For the interface between phases $\alpha$ and $\beta$, the following equation for the macro-scale velocities of an $\alpha \beta$-interface is obtained:

$$
\underline{v}_{\alpha \beta}=-\underline{\underline{R}}_{\alpha \beta}^{-1} \cdot\left(\nabla\left(a_{\alpha \beta} \gamma_{\alpha \beta}\right)+a_{\alpha \beta} \Gamma_{\alpha \beta} \underline{g}-a_{\alpha \beta} \Gamma_{\alpha \beta} \frac{\partial H_{\alpha \beta}}{\partial S_{\mathrm{w}}} \nabla S_{\mathrm{w}}\right) .
$$

$H_{\alpha}$ and $H_{\alpha \beta}$ are the Helmholtz free energies of phases and interfaces, respectively, $a_{\alpha \beta}$ is the specific interfacial area of the $\alpha \beta$-interfaces (i.e., the interfacial area per unit volume), $\gamma_{\alpha \beta}$ is the macro-scale interfacial tension, and $\Gamma_{\alpha \beta}$ is the areal mass density (mass per unit area) of $\alpha \beta$-interfaces.

For the full derivation of Eqs. 8 and 9, we refer to the original work by Hassanizadeh and Gray (1990). A similar result for Eq. 8 was found by Bowen (1982) in the frame of mixture theory.

Another important result of the work of Hassanizadeh and Gray (1990) is the definition of macro-scale capillary pressure. From the residual entropy inequality, it was derived that macro-scale capillary pressure corresponds to the changes in free energies of phases and interfaces due to a change in saturation:

$$
p_{\mathrm{c}}=\rho_{\mathrm{n}} S_{\mathrm{n}} \frac{\partial H_{\mathrm{n}}}{\partial S_{\mathrm{n}}}-\rho_{\mathrm{w}} S_{\mathrm{w}} \frac{\partial H_{\mathrm{w}}}{\partial S_{\mathrm{w}}}-\sum_{\alpha \beta}\left(\frac{\Gamma_{\alpha \beta} a_{\alpha \beta}}{\phi} \frac{\partial H_{\alpha \beta}}{\partial S_{\mathrm{w}}}\right) .
$$

Thermodynamically based "Darcy's Law" without Interfacial Area When interfaces are not included in the derivation of flow equations, no balance equations for interfaces can be formulated and we are left with the equations for the velocities $\underline{v}_{\alpha}$ of the $w$-phase and the $n$-phase as given in Eq. 8. Without accounting for interfaces, the definition of capillary pressure identified in Eq. 10 will simplify to

$$
p_{\mathrm{c}}=\rho_{\mathrm{n}} S_{\mathrm{n}} \frac{\partial H_{\mathrm{n}}}{\partial S_{\mathrm{n}}}-\rho_{\mathrm{w}} S_{\mathrm{w}} \frac{\partial H_{\mathrm{w}}}{\partial S_{\mathrm{w}}} .
$$

Thermodynamically based "Darcy's Law” with Fluid-Fluid Interfacial Area With wninterfaces, in addition to the equation for the velocity of fluid phases given by Eq. 8, we have to account for velocity of the $w n$-interface as given in Eq. 9. Capillary pressure is then given by Eq. 10 as

$$
p_{\mathrm{c}}=\rho_{\mathrm{n}} S_{\mathrm{n}} \frac{\partial H_{\mathrm{n}}}{\partial S_{\mathrm{n}}}-\rho_{\mathrm{w}} S_{\mathrm{w}} \frac{\partial H_{\mathrm{w}}}{\partial S_{\mathrm{w}}}-\frac{\Gamma_{w n} a_{w n}}{\phi} \frac{\partial H_{w n}}{\partial S_{\mathrm{w}}} .
$$

Fluid-solid interfaces could also be included, but are generally of secondary importance compared to fluid-fluid interfaces and therefore not accounted for in this work. The reason for that 
is that the sum of the $w s$ - and $n s$-interfaces is constant (equal to the specific solid surface). The experimental evidence that hysteresis in the capillary pressure-saturation relationship disappears to within the measurement error through inclusion of fluid-fluid interfacial area Chen et al. (2007) only further supports this assumption.

\section{Direct Comparison of Darcy's Law with the Thermodynamically Consistent Approach}

In the next step, we compare the classical two-phase Darcy approach to the thermodynamically consistent theory. By demanding equality of the "Darcy velocities," i.e., the phase fluxes, for the two-phase Darcy approach in Eq. 4 and the thermodynamically consistent approach in Eq. 8, we must have

$$
-\frac{k_{r \alpha} \underline{\underline{K}}}{\mu_{\alpha}} \cdot\left(\nabla p_{\alpha}-\rho_{\alpha} \underline{g}\right)=-\frac{S_{\alpha}^{2} \underline{\underline{K}}_{\alpha}^{*}}{\mu_{\alpha}} \cdot\left(\nabla p_{\alpha}-\rho_{\alpha} \underline{g}+\rho_{\alpha} \frac{\partial H_{\alpha}}{\partial S_{\alpha}} \nabla S_{\alpha}\right) .
$$

Equation 13 is the central part of our comparison and is investigated in detail. The goal of the comparison is to identify if the extended Darcy's law can capture the same physics as the thermodynamically consistent approach and if properties and relationships from the classical approach can be "translated" to and used in the thermodynamically consistent approach. Therefore, we consider the following two cases:

1. The first possibility is to set $k_{r \alpha}=k_{r \alpha}\left(S_{\alpha}\right)$ and also allow that $\underline{K}_{\alpha}^{*}$ depends on saturation $S_{\alpha}$. Note that in this case, $\underline{\underline{K}}_{\alpha}^{*}\left(S_{\alpha}\right)$ is a tensorial quantity which suggests that relative permeability which, in the classical two-phase flow approach is assumed to be a scalar, may be a tensor (Lake 1989; Ataie-Ashtiani et al. 2002). For simplicity, we accept the choice of scalar relative permeabilities in the following.

Demanding that Eq. 13 holds both for $\nabla S_{\alpha}=0$ and $\nabla S_{\alpha} \neq 0$, we find an unresolvable inconsistency in the definition of $p_{\mathrm{c}}$ if interfacial area is not included. By accounting for interfacial area, this inconsistency can be resolved.

2. The second possibility is that $k_{r \alpha}$ is a function of both $S_{\alpha}$ and $\nabla S_{\alpha}$ which implies a more complex material relation for relative permeability than is commonly assumed and measured.

These two possibilities are investigated in more detail in the following subsections.

3.1 Case 1: $\underline{\underline{K}}_{\alpha}^{*}=\underline{\underline{K}}_{\alpha}^{*}\left(S_{\alpha}\right)$ and $k_{r \alpha}=k_{r \alpha}\left(S_{\alpha}\right)$ leading to $\partial H_{\alpha} / \partial S_{\alpha}=0$

As constitutive relationships have to hold independent of gradients in saturation, we can directly deduce a connection between $\underline{\underline{K}}_{\alpha}^{*}\left(S_{\alpha}\right)$ and the relative permeability-saturation relationship of the classical approach for the case $\nabla S_{\alpha}=0$ from Eq. 13. This directly leads to

$$
k_{r \alpha}\left(S_{\alpha}\right) \underline{\underline{K}}=S_{\alpha}^{2} \underline{\underline{K}}_{\alpha}^{*}\left(S_{\alpha}\right) .
$$

But this equation now needs to hold both for the general case with $\nabla S_{\alpha} \neq 0$ (which is the case in unsteady-state flow, for instance) and for the special case of $\nabla S_{\alpha}=0$ (such conditions occur in steady-state flow far away from boundaries; Tiab and Donaldson 2004; Kyte and Rapoport 1958; Cable et al. 2000; Poulsen et al. 2000; Kalam et al. 2007; Dong and Dullien 
1997; Bacri et al. 1990). Inserting Eq. 14 in Eq. 13, we obtain

$$
-\rho_{\alpha} \frac{\partial H_{\alpha}}{\partial S_{\alpha}} \nabla S_{\alpha}=0
$$

For that, of course, we need to restrict considerations to the case where both fluid phases are moving, i.e., where neither $S_{\alpha}$ nor $\underline{K}_{\alpha}^{*}$ are equal to zero. Equation 15 must be valid for both $\nabla S_{\alpha}=0$ and $\nabla S_{\alpha} \neq 0$.

For Eq. 15 to hold for all $\nabla S_{\alpha}$, one must have $\partial H_{\alpha} / \partial S_{\alpha}=0$. This leads to different results depending on whether we include interfacial areas or not:

- No consideration of interfacial area Using the definition of $p_{\mathrm{c}}$ from Eq. 11, we directly obtain $p_{\mathrm{c}}=0$ which is an inconsistency.

- Inclusion of interfacial area Let us recall Eq. 9 where the macro-scale velocity of a $w n$-interface is given by

$$
-\nabla\left(\gamma_{w n} a_{w n}\right)-\Gamma_{w n} a_{w n} \underline{g}+\Gamma_{w n} a_{w n} \frac{\partial H_{w n}}{\partial S_{\mathrm{w}}} \nabla S_{\mathrm{w}}=\underline{\underline{R}}_{w n} \underline{v}_{w n} .
$$

By identifying the term $-\Gamma_{w n} a_{w n}\left(\partial H_{w n} / \partial S_{\mathrm{w}}\right)$ as the product of capillary pressure and porosity, see Eq. 12 with $\partial H_{\alpha} / \partial S_{\alpha}=0$, we obtain

$$
-\phi p_{\mathrm{c}} \nabla S_{\mathrm{w}}=\nabla\left(\gamma_{w n} a_{w n}\right)+\Gamma_{w n} a_{w n} \underline{g}+\underline{\underline{R}}_{w n} \underline{v}_{w n} .
$$

In cases where gravity can be neglected, the equation simplifies to

$$
-\phi p_{\mathrm{c}} \nabla S_{\mathrm{W}}=\nabla\left(\gamma_{w n} a_{w n}\right)+\underline{\underline{R}}_{w n} \underline{v}_{w n} .
$$

The inclusion of interfaces clearly leads to physically meaningful results. If there is a saturation gradient, this can lead to two situations:

1. if interfaces are not moving, gradients in saturation can be balanced either by gravity and/or by gradients in specific interfacial area and/or

2. a saturation gradient may lead to a movement of interfaces (expressed by $\underline{v}_{w n}$ ).

In case the saturation gradient vanishes, flow of interfaces is driven by a gradient in specific interfacial area (if macroscopic interfacial tension is constant). The most important implication of this case is that capillary pressure being defined as $-\frac{\Gamma_{w n} a_{w n}}{\phi} \frac{\partial H_{w n}}{\partial S_{\mathrm{w}}}$ shows that $p_{\mathrm{c}}$ must depend on saturation and specific interfacial area, i.e., $p_{\mathrm{c}}=p_{\mathrm{c}}\left(S_{\mathrm{w}}, a_{w n}\right)$.

\subsection{Case 2: $\partial H_{\alpha} / \partial S_{\alpha} \neq 0$ leading to $k_{r \alpha}=k_{r \alpha}\left(S_{\alpha}, \nabla S_{\alpha}\right)$}

A non-vanishing term $\partial H_{\alpha} / \partial S_{\alpha}$ is "lumped" into the $k_{r \alpha}$ relationship and as a consequence, $k_{r \alpha}=k_{r \alpha}\left(S_{\alpha}, \nabla S_{\alpha}\right)$ in general (only for the special case of $\nabla S_{\alpha}=0, k_{r \alpha}=k_{r \alpha}\left(S_{\alpha}\right)$ ). The dependency on $S_{\alpha}$ and $\nabla S_{\alpha}$ would imply a much more complex material behavior than is commonly assumed and determined experimentally.

Note that the theory does not give any prediction about the magnitude of the terms $\partial H_{\alpha} / \partial S_{\alpha}$ and $\Gamma_{w n} a_{w n}\left(\partial H_{w n} / \partial S_{\mathrm{w}}\right)$; their magnitude has to be determined for a given situation, e.g., in an experiment.

There is indeed experimental evidence that relative permeabilities depends on the capillary number $N_{\mathrm{c}}=\mu v / \sigma$ (Boom et al. 1995, 1996; Avraam and Payatakes 1995b; Henderson et al. 1996, 2000; Skauge et al. 2001; Bartley and Ruth 2001; Ataie-Ashtiani et al. 2002; Tsakiroglou et al. 2004; Li et al. 2005) which was tested by variation of all three parameters 
(viscosity $\mu$, flow velocity $v$, and the interfacial tension $\sigma$ ). The dependency on $v$ leads to an implicit dependency on pressure gradients and thus, saturation gradients in the general case.

Recently, a modified Richards equation for unsaturated flow was proposed by CuetoFelgueroso and Juanes (2009), who introduced a term with a fourth-order saturation gradient in the flow equation. This modification was based on a phase field model, with the aim of describing a flow front instability during infiltration of water into (partially) dry soil. In their formulation, the water flux depends on the third-order gradient of saturation. So, when lumping the relative permeability and this higher-order term into an effective relative permeability, which would not only depend on saturation $S_{\mathrm{w}}$, but also on a third-order gradient of $S_{\mathrm{w}}$.

We will continue by discussing whether Case 1 or 2 is more realistic.

\subsection{Discussion: Magnitude/Importance of the $\partial H_{\alpha} / \partial S_{\alpha}$ Terms}

We have identified two different possibilities where the classical approach and the interfacialarea-based approach provide the same results. From a mathematical point of view, the difference between these two possibilities lies in the question whether the derivatives $\frac{\partial H_{\alpha}}{\partial S_{\alpha}}$ can be assumed to be identical to zero (or negligibly small). Therefore, we will have a closer look at this issue in the following.

We closely follow the line of thought of two publications, Morrow (1970) and Seth and Morrow (2007) who studied the change in the surface area in a pore-scale fluid configuration (in particular pendular rings) and the relation to external work done to the system, e.g., work of drainage of the wetting phase. In Morrow (1970), the reversible pore-scale fluid displacement is studied in an idealized setup assuming that changes in the surface area are only due to external work done on the system.

For isothermal flow, the change in the total Helmholtz free energy of the system is given by

$$
\sum_{\alpha} \frac{\partial H_{\alpha}}{\partial V_{\alpha}} d V_{\alpha}=-\sum_{\alpha=w, n, s} p_{\alpha} d V_{\alpha}^{\prime}+\sum_{\alpha \beta=w n, w s, n s} \sigma_{\alpha \beta} d A_{\alpha \beta},
$$

where $V$ is volume, $\sigma$ is the pore-scale interfacial tension, and $A$ is area. From there, by considering a virtual displacement (implying $\sum_{\alpha} \frac{\partial H_{\alpha}}{\partial V_{\alpha}} d V_{\alpha}=0$ ) and assuming incompressible phases, one obtains

$$
\left(p_{\mathrm{n}}-p_{\mathrm{w}}\right) d V_{\mathrm{w}}+\sum_{\alpha \beta=w n, w s, n s} \sigma_{\alpha \beta} d A_{\alpha \beta}=0 .
$$

Dividing this equation by $d V_{\mathrm{w}}$, multiplying both sides by $\phi$, taking account of $\sigma_{\alpha \beta}=$ $-a_{\alpha \beta} \Gamma_{\alpha \beta} \frac{\partial H_{\alpha \beta}}{\partial a_{\alpha \beta}}$ and $\phi \cdot \frac{\partial A_{\alpha \beta}}{\partial V_{\mathrm{w}}}=\frac{\partial a_{\alpha \beta}}{\partial S_{\mathrm{w}}}$ yields

$$
\phi\left(p_{\mathrm{n}}-p_{\mathrm{w}}\right)=\sum_{\alpha \beta=w n, w s, n s} a_{\alpha \beta} \Gamma_{\alpha \beta} \frac{\partial H_{\alpha \beta}}{\partial a_{\alpha \beta}} \frac{\partial a_{\alpha \beta}}{\partial S_{\mathrm{w}}} .
$$

The term $\frac{\partial H_{\alpha \beta}}{\partial a_{\alpha \beta}} \frac{\partial a_{\alpha \beta}}{\partial S_{\mathrm{w}}}$ can be transformed making use of the equality

$$
d H_{\alpha \beta}=\left.\frac{\partial H_{\alpha \beta}}{\partial a_{\alpha \beta}}\right|_{S_{\mathrm{w}}} d a_{\alpha \beta}+\left.\frac{\partial H_{\alpha \beta}}{\partial S_{w}}\right|_{a_{\alpha \beta}} d S_{\mathrm{w}},
$$


considering the case $d H_{\alpha \beta}=0$ and dividing by $d S_{\mathrm{w}}$ which yields

$$
\frac{\partial H_{\alpha \beta}}{\partial a_{\alpha \beta}} \frac{\partial a_{\alpha \beta}}{\partial S_{\mathrm{w}}}=-\frac{\partial H_{\alpha \beta}}{\partial S_{\mathrm{w}}}
$$

and finally

$$
\phi\left(p_{\mathrm{n}}-p_{\mathrm{w}}\right)=-\sum_{\alpha \beta=w n, w s, n s} a_{\alpha \beta} \Gamma_{\alpha \beta} \frac{\partial H_{\alpha \beta}}{\partial S_{\mathrm{w}}},
$$

which is identical to our result for $p_{\mathrm{c}}$ from Eq. 12 for $\frac{\partial H_{\alpha}}{\partial S_{\alpha}}=0$ and considering $w n$-interfaces only (Case 1), i.e., $p_{\mathrm{c}}=-\frac{\Gamma_{w n} a_{w n}}{\phi} \frac{\partial H_{w n}}{\partial S_{\alpha}}$.

Note that the terms $\frac{\partial H_{\alpha}}{\partial S_{\alpha}}$ vanish in Morrow's work only due to the assumption of incompressibility and reversible displacement, which does not fully describe the general case because of irreversible displacement contributions. In case of irreversible displacement, $\sum_{\alpha} \frac{\partial H_{\alpha}}{\partial V_{\alpha}} d V_{\alpha} \neq 0$ leading to $\frac{\partial H_{\alpha}}{\partial S_{\alpha}} \neq 0$. Indeed, Seth and Morrow (2007) found that Eq. 20 does not hold for irreversible displacement. They found a discrepancy between the work done to the system (left hand side of Eq. 20) and the change in internal energy, i.e., the change in surface free energy (right hand side of Eq. 20). The external work $W$ done to the system is based on the pressure term in Eq. 20,

$$
W=\phi V_{\mathrm{b}} \int p_{\mathrm{c}} d S_{\mathrm{w}}
$$

where $V_{\mathrm{b}}$ is the bulk volume of the solid. The change in internal energy is computed from the change in surface free energy $\Delta H$,

$$
\Delta H=\sigma_{w n}\left(\Delta A_{w n}+\Delta A_{n s} \cos \Theta\right),
$$

where $\Theta$ is the contact angle and account was taken of Young's equation. The ratio of the change in surface free energy over the work done to the system is defined as net efficiency

$$
E_{d}=\frac{\Delta H}{W} .
$$

Seth and Morrow found values of $E_{d}$ between 0.36 and 0.85 which indicates that in the general case (irreversible displacement), the terms $\frac{\partial H_{\alpha}}{\partial S_{\alpha}}$ have significant contributions and cannot be assumed to be small.

As pointed out in Case 2, $\partial H_{\alpha} / \partial S_{\alpha} \neq 0$ leads to $k_{r \alpha}=k_{r \alpha}\left(S_{\alpha}, \nabla S_{\alpha}\right)$, i.e., $k_{r, \alpha}$ can be different for $\nabla S_{\mathrm{w}}=0$ and $\nabla S_{\mathrm{w}} \neq 0$. In most field situations, e.g., in oil recovery, there is unsteady-state flow where saturations change over time and space, i.e., saturation gradients $\nabla S_{\mathrm{w}} \neq 0$ occur in particular in the case of moving fronts (Buckley and Leverett 1942). Since saturation changes are typically a combination of reversible and irreversible processes, for the irreversible part, the $\partial H_{\alpha} / \partial S_{\alpha}$ terms do not vanish: they can be of relevant magnitude and are lumped into the $k_{r \alpha}=k_{r \alpha}\left(S_{\alpha}, \nabla S_{\alpha}\right)$ relationships.

In laboratory experiments, however, relative permeability $k_{r \alpha}$ is typically determined in both unsteady-state and in steady-state flow experiments. For steady-state flow, saturations do not change over time. The overwhelming majority of experimental studies including numerical simulations (Lake 1989; Tiab and Donaldson 2004; Langaas et al. 1996; Chen and Wood 2001; Cable et al. 2000; Urkedal et al. 2000) show that in steady-state experiments, apart from sample heterogeneity and end-effects (Huang and Honarpour 1996), the saturation gradients are small, i.e., $\nabla S_{\mathrm{w}} \approx 0$. Under such conditions, the $\partial H_{\alpha} / \partial S_{\alpha} \nabla S_{\alpha}$ terms vanish. This would imply that relative permeability determined in steady-state flow and in unsteady-state flow 
are not necessarily identical which is supported by experimental evidence (Tsakiroglou et al. 2004).

\section{Conclusions}

In this work, we have compared the classical model for two-phase flow with an existing thermodynamically consistent approach. By demanding the equality of the specific fluxes of both approaches (Darcy velocities), we could show that the extended two-phase Darcy's law is compatible with the thermodynamically consistent approach if either (i) relative permeabilities are a function of saturation only, but capillary pressure depends on saturation and specific interfacial area or (ii) relative permeabilities are a function of saturation and saturation gradients. Significant irreversible contributions to the work of drainage suggest that the latter is the more likely case. It implies a more complex material behavior than commonly assumed. The sensitivity of relative permeability with respect to saturation gradients must be determined experimentally, but theoretical considerations suggest that the sensitivity may be low in case of reversible displacement, but significant in the general case (irreversible displacement). While this comparison study provided insights on the conditions under which the classical and the thermodynamically based approach provide the same results, it needs to be stressed that it is desirable to describe two-phase flow by explicitly accounting for the driving forces for flow (as is done in the thermodynamically based approach) rather than adapt constitutive functions.

In summary, we have demonstrated that under flow conditions, the classical two-phase flow approach can be "mapped" onto the thermodynamically consistent flow theory by Hassanizadeh and Gray (1990) when either interfacial area and/or a more complex relationship for relative permeability are introduced. The direct comparison between both theories shows a route how experimental data collected in the framework of two-phase Darcy flow can be "translated" to determine the magnitude of the parameters in the thermodynamically consistent theory.

Note that while mapping under flow conditions is possible, there are clear differences between both theories under no-flow conditions. The difference is whether pressure gradients have to be zero (classical two-phase flow approach) or may be non-zero (thermodynamically consistent theory). We currently investigate this issue experimentally.

Acknowledgments The authors acknowledge A. W. Cense, J. G. Maas, W. Scherpenisse, P. Doe, and J. Jennings from Shell for their helpful discussions. J. Niessner and S.M. Hassanizadeh are members of the International Research Training Group NUPUS, financed by the German Research Foundation (DFG) and The Netherlands Organisation for Scientific Research (NWO), and thank the DFG (GRK 1398) and NWO/ALW for their support.

Open Access This article is distributed under the terms of the Creative Commons Attribution Noncommercial License which permits any noncommercial use, distribution, and reproduction in any medium, provided the original author(s) and source are credited.

\section{References}

Ataie-Ashtiani, B., Hassanizadeh, S.M., Celia, M.A.: Effects of heterogeneities on capillary pressuresaturation-relative permeability relationships. J. Contam. Hydrol. 56, 175-192 (2002)

Auriault, J.: Transport in Porous Media: Upscaling by Multiscale Asymptotic Expansions, pp. 3-56. Springer, Berlin (2005) 
Avraam, D., Payatakes, A.: Flow regimes and relative permeabilities during steady-state two-phase flow in porous media. J. Fluid Mech. Digit. Arch. 293, 207-236 (1995)

Ayub, M., Bentsen, R.G.: Interfacial viscous coupling: a myth or reality? J. Petrol Sci. Eng. 23, 13-29 (1999)

Ayub, M., Bentsen, R.G.: Experimental testing of interfacial coupling in two-phase flow in porous media. Pet. Sci. Technol. 23, 863-897 (2005)

Bacri, J.C., Rosen, M., Salin, D.: Capillary hyperdiffusion as a test of wettability. Europhys. Lett. 2(2), 127132 (1990)

Bartley, J.T., Ruth, D.W.: Experimental investigation of unsteady-state relative permeability in sand-packs. Society of Core Analysis Conference Paper SCA, vol. 22, pp.1-14 (2001)

Bear, J., Bachmat, Y.: Introduction to Modeling of Transport Phenomena in Porous Media. Kluwer Academic Publishers, The Netherlands (1990)

Berg, S., Cense, A.W., Hofman, J.P., Smits, R.M.M.: Two-phase flow in porous media with slip boundary condition. Transp. Porous Media 74(3), 275-292 (2008)

Boom, W., Wit, K., Schulte, A.M., Oedai, S., Zeelenberg, J.P.W., Maas, J.G.: Experimental evidence for improved condensate mobility at near-wellbore flow conditions. Society of Petroleum Engineers (SPE 30766):667-675 (1995)

Boom, W., Wit, K., Zeelenberg, J.P.W., Weeda, H.C., Maas, J.G.: On the use of model experiments for assessing improved gas-condensate mobility under near-wellbore flow conditions. Society of Petroleum Engineers (SPE 36714):343-353 (1996)

Bottero, S., Hassanizadeh, S., Kleingeld, P., Bezuijen A.: Experimental study of dynamic capillary pressure effect in two-phase flow in porous media. In: Proceedings of the XVI International Conference on Computational Methods in Water Resources (CMWR), Copenhagen, Denmark (2006)

Bowen, R.: Compressible porous media models by use of the theory of mixtures. Int. J. Eng. Sci. 20(6), 697-735 (1982)

Brinkman, H.: A calculation of the viscous force exerted by a flowing fluid on a dense swarm of particles. Appl. Sci. Res. A1, 27-33 (1947)

Buckley, S.E., Leverett, M.C.: Mechanism of fluid displacements in sands. Trans. AIME 146, 107-116 (1942)

Cable, A., Mott, R., Spearing, M.: X-ray in-situ saturation in gas condensate relative permeability studies. Society of Core Analysis Conference Paper SCA 2000-39, pp. 1-13 (2000)

Chen, A.L., Wood, A.C.: Rate effects on water-oil relative permeability. Society of Core Analysis Conference Paper SCA 2001-19, pp. 1-12 (2001)

Chen, D., Pyrak-Nolte, L.J., Griffin, J., Giordano, N.J.: Measurement of interfacial area per volume for drainage and imbibition. Water Resour. Res. 43, W12504 (2007)

Cueto-Felgueroso, L., Juanes, R.: A phase field model of unsaturated flow. Water Resour. Res. 43, W10-409 (2009)

Culligan, K., Wildenschild, D., Christensen, B., Gray, W., Rivers, M., Tompson, A.: Interfacial area measurements for unsaturated flow through a porous medium. Water Resour. Res. 40, 1-12 (2004)

Culligan, K.A., Wildenschild, D., Christensen, B.S.B., Gray, W.G., Rivers, M.L.: Pore-scale characteristics of multiphase flow in porous media: a comparison of air-water and oil-water experiments. Adv. Water Resour. 29, 227-238 (2006)

Dalla, E., Hilpert, M., Miller, C.T.: Computation of the interfacial area for two-fluid porous media systems. J. Contam. Hydrol. 56, 25-48 (2002)

Darcy, H.: Détermination des lois d'écoulement de l'eau à travers le sable. In: Victor Dalmont P (ed.) Les Fontaines Publiques de la Ville de Dijon, pp. 590-594. V. Dalmont, Paris (1856)

Das, D.B., Gauldie, R., Mirzaei, M.: Dynamic effects for two-phase flow in porous media: fluid property effects. AIChE J. 53(10), 2505-2520 (2007)

Di Carlo, D.: Experimental measurements of saturation overshoot on infiltration. Water Resour. Res. 40, W04215 (2004)

Di Carlo, D.: Modeling observed saturation overshoot with continuum additions to standard unsaturated theory. Adv. Water Resour. 28(10), 1021-1027 (2005)

Dong, M., Dullien, F.A.L.: A new model for immiscible displacement in porous media. Transp. Porous Media 27, 185-204 (1997)

Dullien, F.A.L.: Porous Media-Fluid Transport and Pore Structure, 2nd edn. Academic Press, New York (1992)

Eastwood, J.E., Spanos, T.J.T.: Steady-state contercurrent flow in one dimension. Transp. Porous Media 6, 173-182 (1991)

Gladkikh, M., Bryant, S.: Prediction of interfacial areas during imbibition in simple porous media. Adv. Water Resour. 26, 609-622 (2003)

Gray, W., Hassanizadeh, S.: Averaging theorems and averaged equations for transport of interface properties in multiphase systems. Int. J. Multi-Phase Flow 15, 81-95 (1989) 
Gray, W., Miller, C.: Thermodynamically constrained averaging theory approach for modeling of flow in porous media: 1. Motivation and overview. Adv. Water Resour. 28(2), 161-180 (2005)

Hassanizadeh, S.M., Gray, W.G.: General conservation equations for multi-phase systems: 3. Constitutive theory for porous media flow. Adv. Water Resour. 3, 25-40 (1980)

Hassanizadeh, S.M., Gray, W.G.: High velocity flow in porous media. Transp. Porous Media 2, 521-531 (1987)

Hassanizadeh, S.M., Gray, W.G.: Mechanics and thermodynamics of multiphase flow in porous media including interphase boundaries. Adv. Water Resour. 13(4), 169-186 (1990)

Hassanizadeh, S.M., Gray, W.G.: Thermodynamic basis of capillary pressure in porous media. Water Resour. Res. 29(10), 3389-3405 (1993a)

Hassanizadeh, S.M., Gray, W.G.: Toward an improved description of the physics of two-phase flow. Adv. Water Resour. 16(1), 53-67 (1993b)

Hassanizadeh, S.M., Celia, M.A., Dahle, H.K.: Dynamic effect in capillary pressure-saturation relationship and its impact on unsaturated flow. Vadose Zone J. 1, 38-57 (2002)

Henderson, G.D., Danesh, A., Teharani, D.H., Al-Shaidi, S., Peden, J.M.: Measurement and correlation of gas condensate relative permeability by the steady-state method. SPE J. 1(2), 191-201 (1996)

Henderson, G.D., Danesh, A., Al-kharusi, B., Tehrani, D.: Generating reliable gas condensate relative permeability data used to develop a correlation with capillary number. J. Pet. Sci. Eng. 25, 79-91 (2000)

Huang, D.D., Honarpour, M.M.: Capillary end effects in coreflood calculations. Society of Core Analysis Conference Paper SCA 96-34, pp. 1-10 (1996)

Huang, H., Lu, Xy.: Relative permeabilities and coupling effects in steady-state gas-liquid flow in porous media: a lattice boltzmann study. Phys. Fluids 21, 092-104 (2009)

Huyghe, J., Oomens, C., Van Campen, D., Heethaar, R.: Low reynolds number steady state flow through a branching network of rigid vessels: I. A mixture model. Biorheology 26, 73-84 (1989)

Jackson, A., Miller, C., Gray, W.: Thermodynamically constrained averaging theory approach for modeling flow and transport phenomena in porous medium systems: 6. Two-fluid-phase flow. Adv. Water Resour. 32(6), 779-795 (2009)

Joekar-Niasar, V., Hassanizadeh, S., Leijnse, A.: Insights into the relationship among capillary pressure, saturation, interfacial area and relative permeability using pore-scale network modeling. Transp. Porous Media. 74(2), 201-219 (2008)

Joekar-Niasar, V., Hassanizadeh, S., Pyrak-Nolte, L., Berentsen, C.: Simulating drainage and imbibition experiments in a high-porosity micro-model using an unstructured pore-network model. Water Resour. Res. 45, W02430 (2009). doi:10.1029/2007WR006641

Kalam, Z., Obeida, T., Al Masaabi, A.: Acceptable water-oil and gas-oil relative permeability measurements for use in reservoir simulation models. Society of Core Analysis Conference Paper SCA. 11, pp. 1-12 (2007)

Kalaydjian, F.: A macroscopic description of multiphase flow in porous media involving spacetime evolution of fluid/fluid interface. Transp. Porous Media 2, 537-552 (1987)

Killough, J.E.: Reservoir simulation with history dependent saturation functions. Society of Petroleum Engineers SPE 5106 (1976)

Kyte, J.R., Rapoport, L.A.: Linear waterflood behavior and end effects in water-wet porous media. Society of Petroleum Engineers Conference Paper SPE 929 G, pp. 47-50 (1958)

Lake, L.W.: Enhanced Oil Recovery. Prentice Hall, Upper Saddle River, NJ (1989)

Langaas, K., Ekrann, S., Ebeltoft, E.: The impact of using composite cores on core analysis results. Society of Core Analysis Conference Paper SCA 96-02, pp. 1-10 (1996)

Li, H., Pan, C., Miller, C.T.: Pore-scale investigation of viscous coupling effects for two-phase flow in porous media. Phys. Rev. E 72, 026-705 (2005)

Manthey, S., Hassanizadeh, M.S., Helmig, R.: Macro-scale dynamic effects in homogeneous and heterogeneous porous media. Transp. Porous Media 58, 121-145 (2005)

Marle, C.M.: On macroscopic equations governing multiphase flow with diffusion and chemical reactions in porous media. Int. J. Eng. Sci. 20(5), 643-662 (1982)

Miller, C., Gray, W.: Thermodynamically constrained averaging theory approach for modeling flow and transport phenomena in porous medium systems: 2. Foundation. Adv. Water Resour. 28(2), 181-202 (2005)

Mirzaei, M., Das, D.B.: Dynamic effects in capillary pressure-saturations relationships for two-phase flow in 3d porous media: implications of micro-heterogeneities. Chem. Eng. Sci. 62(7), 1927-1947 (2007)

Morrow, N.: Physics and thermodynamics of capillary action in porous media. Ind. Eng. Chem. 62(6) 32-56 (1970)

Niessner, J., Hassanizadeh, S.: A model for two-phase flow in porous media including fluid-fluid interfacial area. Water Resour. Res. 44, W08439. doi:10.1029/2007WR006721 (2008)

Niessner, J., Hassanizadeh, S.: Modeling kinetic interphase mass transfer for two-phase flow in porous media including fluid-fluid interfacial area. Transp. Porous Media 80(2), 329-344 (2009) 
Niessner, J., Hassanizadeh, S.: Non-equilibrium interphase heat and mass transfer during two-phase flow in porous media - theoretical considerations and modeling. Adv. Water Resour. 32(12), 1756-1766 (2009)

Oostrom, M., White, M.D., Brusseau, M.L.: Theoretical estimation of free and entrapped nonwetting-wetting fluid interfacial areas in porous media. Adv. Water Resour. 24, 887-898 (2001)

Poulsen, S., Skauge, T., Dyrhol, S.O., Skauge, E. Stenby, A.: Including capillary pressure in simulations of steady state relative permeability experiments. Society of Core Analysis Conference Paper SCA 2000-14, pp. 1-12 (2000)

Seth, S., Morrow, N.R.: Efficiency of the conversion of work of drainage to surface energy for sandstone and carbonate. SPE Reserv. Eval. Eng. SPE 102490, 338-347 (2007)

Skauge, A., Thorsen, T., Sylte, A.: Rate selection for waterflooding of intermediate wet cores. Society of Core Analysis Conference Paper SCA 2001-20, pp. 1-14 (2001)

Stauffer, F.: Time dependence of the relations between capillary pressure, water content and conductivity during drainage of porous media. In: On scale effects in porous media, IAHR, Thessaloniki, Greece (1978)

Tiab, D., Donaldson, E.C.: Petrophysics: Theory and Practice of Measuring Reservoir Rock and Fluid Transport Properties, 2nd edn. Gulf Professional Publishing, Burlington, MA (2004)

Tsakiroglou, C.D., Avraam, D.G., Payatakes, A.C.: Simulation of the immiscible displacement in porous media using capillary pressure and relative permeability curves from transient and steady-state experiments. Society of Core Analysis Conference Paper SCA 2004-12, pp. 1-13 (2004)

Urkedal, H., Ebeltoft, E., Nordtvedt, J.E., Watson, A.T.U.: A new design of steady-state type experiments for simultaneous estimation of two-phase flow functions. SPE Reserv. Eng. 3(3), 230-238 (2000)

Vankan, W., Huyghe, J., Janssen, J., Huson, A.: Poroelasticity of satured solids with an application to blood perfusion. Int. J. Eng. Sci. 34, 1019-1031 (1996)

Zhang, X.Y., Bentsen, R.G., Cunha, L.B.: Investigations of interfacial coupling phenomena and its impact on recovery factor. J. Can. Pet. Technol. 47(7), 26-32 (2008) 